
FciDioc
International Journal of Cardiology and Research (IJCRR)

ISSN 2470-4563

\title{
A Study on Patients Presenting Like Acute Coronary Syndrome in the Absence of Obstructive Coronary Artery Disease on Coronary Angiogram
}

Research Article

Basu $\mathrm{S}^{1}$, Majumder $\mathrm{B}^{2}$, Sarkar $\mathrm{R}^{3 *}$, Mazumdar $\mathrm{J}^{4}$

${ }^{1}$ Assistant Profeesor, Department of Cardiology, North Bengal Medical College, India.

${ }^{2}$ Associate professor, Department of Cardiology, R.G.Kar Medical College, India.

${ }^{3}$ R G Kar Medical College, West Bengal University of Health Sciences, India.

${ }^{4}$ Resident in Cardiology Department.

\section{Abstract}

Introduction: Particular interest has been raised in mechanisms of acute coronary syndromes (ACS) that cannot be explained by the presence of obstructive coronary atherosclerosis. Our study strived to find the prevalence, clinical profile, etiology and short term prognosis amongst patients without significant obstructive coronary artery disease (CAD) presenting like ACS to a tertiary care hospital of Eastern India.

Methods: The study was a hospital based observational, descriptive longitudinal study on 968 patients with chest pain suggestive of ACS at the time of presentation.

Result: Among the consecutive 968 patients, 63 were found to have non-obstructive lesion in coronary angiography (CAG), of which $23.8 \%$ were with normal coronaries and $76.2 \%$ with minor/mild CAD. Except for hypertension, there was no significant difference in prevalence of the conventional risk factors like smoking, dyslipidaemia, and diabetes mellitus between these two groups. Coronary vasospasm $(9 \%)$, myocardial bridge $(8 \%)$, spontaneous dissection and ectasia $(3 \%$ each) and abnormal origin $(2 \%)$ were the other findings. Slow flow was most common $(68 \%)$ in STEMI subgroup. The Kaplan - Meier survival curve analysis showed significant difference in terms of MACE free survival $(p=0.04)$ between two groups but not in terms of overall death $(\mathrm{p}=0.68)$. Univariate analysis showed regional wall motion abnormality and arrhythmia as significant predictor of major adverse cardiac events (MACE) remains.

Conclusion: Non - obstructive CAD in patients with ACS remains uncommon finding. The conventional risk factors are not significantly different in subgroup analysis except hypertension and there was significant difference in terms of MACE free survival in these patients.

Keywords: Non-Obstructive Coronary Artery Disease; Acute Coronary Syndrome; Coronary Angiogram; Regional Wall Motion Abnormality; Cardiac Biomarkers.

Abbreviations: ACS: Acute Coronary Syndromes; CAD: Coronary Artery Disease; CAG: Coronary Angiography; CSFP: Coronary Slow Flow Phenomenon; MACE: Major Adverse Cardiac Events; IHD: Ischemic Heart Disease; RWMA: Regional Wall Motion Abnormality; CKD: Chronic Kidney Disease; MINOCA: Myocardial Infarction With Non-obstructive Coronary Arteries; NObCAD: Non-Obstructive Coronary Artery Disease; ObCAD: Obstructive Coronary Artery Disease.

\section{Introduction}

Ischemic heart disease (IHD) is leading cause of mortality due to Cardiovascular disease worldwide [1] and acute coronary syndromes (ACS) are the commonest causes of mortality in patients with CAD. In more recent years, particular interest has been raised by the causes and mechanisms of acute coronary syndromes (ACS) that cannot be explained by the presence of obstructive coronary atherosclerosis. Non-obstructive coronary artery disease (CAD) is atherosclerotic plaque that would not be expected to obstruct blood flow or result in anginal symptoms. Although such lesions are relatively common, occurring in $10 \%$

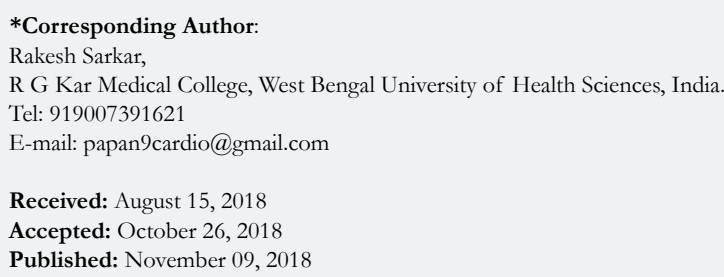

Citation: Sarkar R, Basu S. A Study on Patients Presenting Like Acute Coronary Syndrome in the Absence of Obstructive Coronary Artery Disease on Coronary Angiogram. Int J Cardiol Res. 2018;5(5):131-136. doi: http://dx.doi.org/10.19070/2470-4563-1800022

Copyright: Rakesh Sarkar ${ }^{\circ}$ 2018. This is an open-access article distributed under the terms of the Creative Commons Attribution License, which permits unrestricted use, distribution and reproduction in any medium, provided the original author and source are credited. 
to $25 \%$ of patients undergoing coronary angiography [2, 3$]$ their presence has been characterized as - insignificant or - no significant CAD in the medical literature. Patients with ACS and unobstructed coronary arteries represent a clinical dilemma and their management is quite variable in current practice. Despite the prevalence of non-obstructive CAD identified by coronary angiography, little is known about its risk of adverse outcomes. So this study is aimed at finding out the incidence prevalence of non obstructive coronary artery disease in a tertiary health care centre like our medical college with evaluation of their disease characteristics, the prognostic implication after advocating treatment with the limited knowledge of treating such patients.

\section{Objective}

The patients without significant obstructive CAD presenting like ACS is a heterogeneous population etiologically and thus difficult to be treated in a single management guideline as there is paucity of study in this regard. The objective of our study was to find out.

1. The prevalence of normal coronary angiogram among patients presenting with ACS,

2. The clinical profile and etiology of the ACS like presentation among these patients and

3. The short term prognosis of these patients.

\section{Methods}

One year, from August 2015 to August 2016, Hospital based Observational, prospective study on 968 patients with chest pain clinically suggestive of ACS defined as 2 of the 3 criteria:

1. Dynamic change in ECG and ECG suggestive of classical STEMI

2. Cardiac markers positive

3. RWMA (Regional wall motion abnormality) in echocardiography.

\section{Exclusion Criteria}

1. Patients with kown coronary artery disease proved by CAG(coronary angiography) previously or detected to have Obstructive CAD.

2. Patients who had been undergone revascularisation prior to this event.

3. Patients with pericarditis and known severe valvular heart disease.

4. Patients with severe respiratory infection.

5. Patients with known chronic inflammatory diseases.

\section{Study Tools}

1. ECG to be done by the available instrument in cardiology department/emergency

2. Echocardiography by machine model no-Siemen Acuson C70

3. coronary angiography by $\mathrm{C}$-arm machine Axiom-Sensis XP

4. Blood parameters to be assessed by available techniques in the hospital

Diagnostic coronary angiogram showing $<50 \%$ occlusion of these patients (if not attempted thrombolysis) are diagnosed as non-obstructive $\mathrm{CAD}$ which were further dichotomized as those with normal CAG (without minimal plaque) and minor CAD (obstruction $<50 \%$ ). The patients were followed up for at least 3 months and upto the possible study tenure.

The study proposal with other relevant documents were submitted to the institutional ethics committee for review and approval. The study was commenced only after such approval was obtained in writing.

The data collected were summarized in Microsoft excel sheets. Only those patients who could be followed up for at least 3 months or those who expired during the study period were included. Patients who were lost to follow-up were excluded. The results were then analyzed in Microsoft Excel (2016), Medcalc 17.1 version. Survival analysis including Kaplan Meier, Log Rank, $\log$ survival distribution function, Hazard Function, Receiveroperating Characteristic curves and Cox Regression models were used and the sensitivity, specificity and level of significance ( $p$ value) of the parameters studied were calculated.

\section{Result}

Among the 968 patients presenting like ACS as diagnosed using the criteria mentioned earlier only $63(6.5 \%)$ was found to have non-obstructive lesion in angiography. The male patients (41) out numbers the female (22) in the study population with the mean age being slightly higher $(44.78 \pm 14.29$ Vs $41.36 \pm$ 13.28) in male group though it was not statistically significant. Among the total population $23.8 \%$ were found to have normal angiography i.e. even without any minor plaque and the rest with minor/mild CAD. The prevalence of the conventional risk factors like smoking, dyslipidemia, diabetes mellitus except hypertension were not significantly different in these two groups (Table 1). Categorically, NSTEMI/UA together constitutes almost $68.9 \%$ of the total study population which indicates that patients presenting like STEMI with non-obstructive coronary artery disease is $<$ one third of the study population. Apart

Table 1. Comparison of risk factors among patients with normal and minor CAD.

\begin{tabular}{|c|c|c|c|c|c|}
\hline & $\begin{array}{c}\text { Normal coronary } \\
(<\mathbf{2 0} \text { \% stenosis })\end{array}$ & $\begin{array}{c}\text { Minor CAD } \\
(\mathbf{2 0 \%} \text { stenosis })\end{array}$ & Odds ratio & $\begin{array}{c}\mathbf{9 5 \%} \text { confidence } \\
\text { interval }\end{array}$ & P value \\
\hline Smoking & $2 / 15$ & $18 / 48$ & 3.900 & $0.7879-19.3034$ & 0.0953 \\
\hline Diabetes mellitus & $0 / 15$ & $10 / 48$ & 8.4545 & $0.4663-153.3019$ & 0.1488 \\
\hline Hypertension & $1 / 15$ & $24 / 48$ & 14 & $1.7038-115.0389$ & 0.014 \\
\hline Dyslipidemia & $2 / 15$ & $14 / 48$ & 2.6765 & $0.5331-13.4381$ & 0.2318 \\
\hline Family history & $1 / 15$ & $10 / 48$ & 3.6842 & $0.4313-31.4724$ & 0.2334 \\
\hline
\end{tabular}


Figure 1. Prevalence of various etiologies of ACS with non-obstructive lesions.

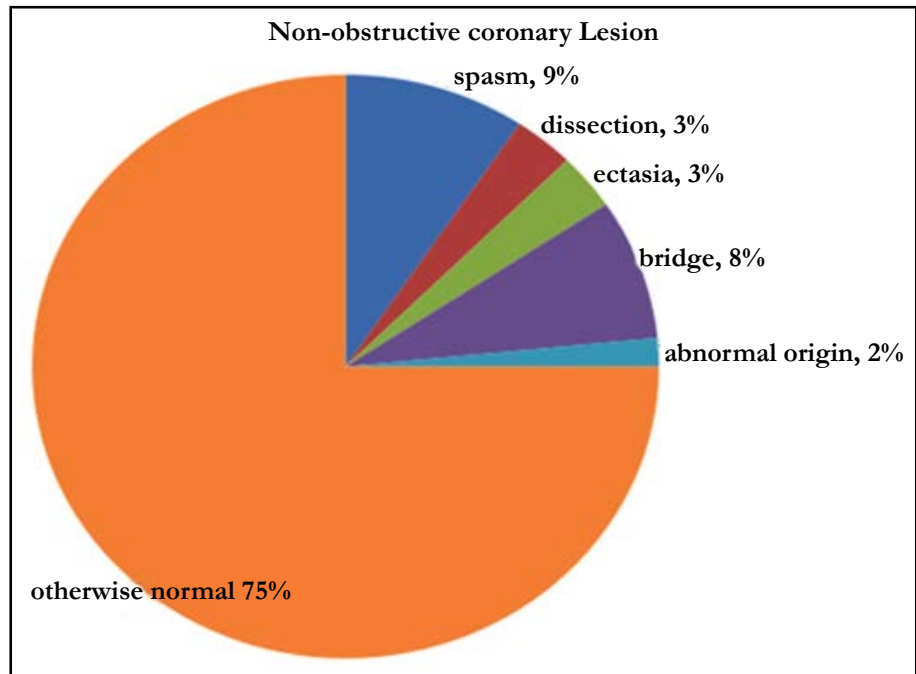

Table 2. Multiple logistic regression analysis of the variables to predict the major adverse cardiovascular events (MACE).

\begin{tabular}{|c|c|c|c|c|c|}
\hline Independent variables & Coefficient & Std. error & $\mathbf{r}_{\text {partial }}$ & $\mathbf{t}$ & $\mathbf{p}$ \\
\hline constant & 1.9491 & & & & \\
\hline CBG & -0.0001699 & 0.001087 & -0.02127 & -0.156 & 0.876 \\
\hline Creatinine & 0.2532 & 0.2342 & 0.1456 & 1.081 & 0.284 \\
\hline EF $\%$ & -0.04277 & 0.007914 & -0.5925 & -5.404 & $<0.0001$ \\
\hline Max stenosis & 0.002249 & 0.004674 & 0.06534 & 0.481 & 0.633 \\
\hline SBP & 0.0007628 & 0.004293 & 0.02417 & 0.178 & 0.8597 \\
\hline DBP & 0.003355 & 0.009976 & 0.04572 & 0.336 & 0.738 \\
\hline Hospital stay & -0.005462 & 0.01258 & -0.05899 & -0.434 & 0.665 \\
\hline
\end{tabular}

Table 3. Predictors of mortality in patients with non-obstructive CAD.

\begin{tabular}{|c|c|c|c|}
\hline Factors & Odds_ratio & $\mathbf{9 5 \%}$ CL & p \\
\hline Biomarker & 5.2 & $0.58-46.05$ & 0.13 \\
\hline Arrhythmia & 33.85 & $1.83-626.22$ & 0.018 \\
\hline Dyspnea & 9.78 & $0.53-179.8$ & 0.12 \\
\hline RWMA & 7.8 & $0.42-143.84$ & 0.16 \\
\hline Slow flow & 2.88 & $0.51-16.13$ & 0.22 \\
\hline H/O fever & 7.5 & $1.30-43.06$ & 0.024 \\
\hline Pericardial effusion & 4.5 & $0.35-57.30$ & 0.24 \\
\hline
\end{tabular}

arrhythmia Slow flow $\begin{aligned} & \text { Pericardia } \\ & \text { effusion }\end{aligned}$

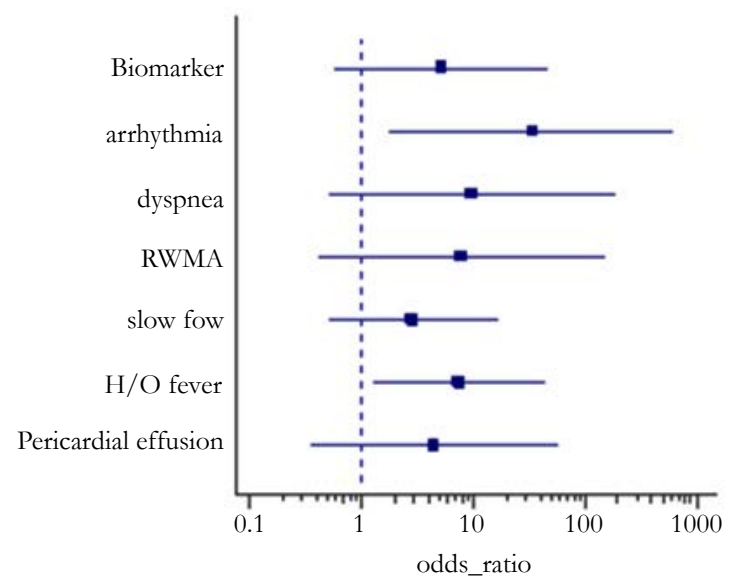

$\ddagger$ Forest plot to compare the Odds ratio of different variables in univariate analysis as the predictor of all cause mortality. 
Table 4. Predictors of adverse outcomes (MACE).

\begin{tabular}{|c|c|c|c|}
\hline Factors & odds_ratio & $\mathbf{9 5 \%} \mathbf{C L}$ & $\mathbf{p}$ \\
\hline Biomarker & 1.42 & $0.47-4.31$ & 0.52 \\
\hline Arrhythmia & 4.57 & $1.45-14.35$ & 0.009 \\
\hline Dyspnea & 1.76 & $0.53-5.78$ & 0.34 \\
\hline RWMA & 5.35 & $1.09-26.13$ & 0.03 \\
\hline Slow flow & 2.25 & $0.74-6.82$ & 0.14 \\
\hline Pericardial effusion & 5.05 & $0.42-59.52$ & 0.19 \\
\hline H/O fever & 1.1 & $0.34-3.52$ & 0.87 \\
\hline \multicolumn{4}{|c}{$\begin{array}{l}\text { Pericardial } \\
\text { effusion }\end{array}$} \\
arrhythmia & Slow flow
\end{tabular}

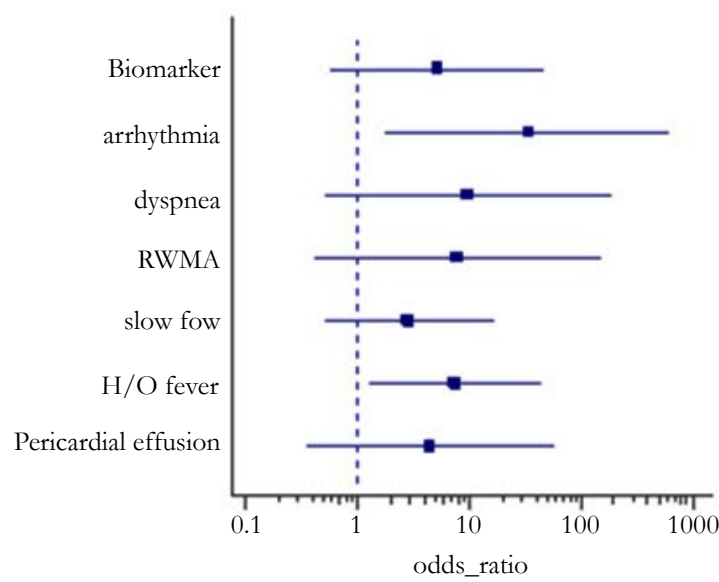

$\ddagger$ Forest plot to compare the Odds ratio of different variables in Univariate analysis as the predictor of MACE. Among above variables, only arrhythmia (OR 4.5714,95\%CI 1.45600 - 14.3535, p value 0.0092) and the RWMA (OR 5.3519, $95 \%$ CI 0.3436-3.5254, p value-0.0382) are significant.

Figure 2. Kaplan Meier survival curve analysis for development of MACE in follow up period.

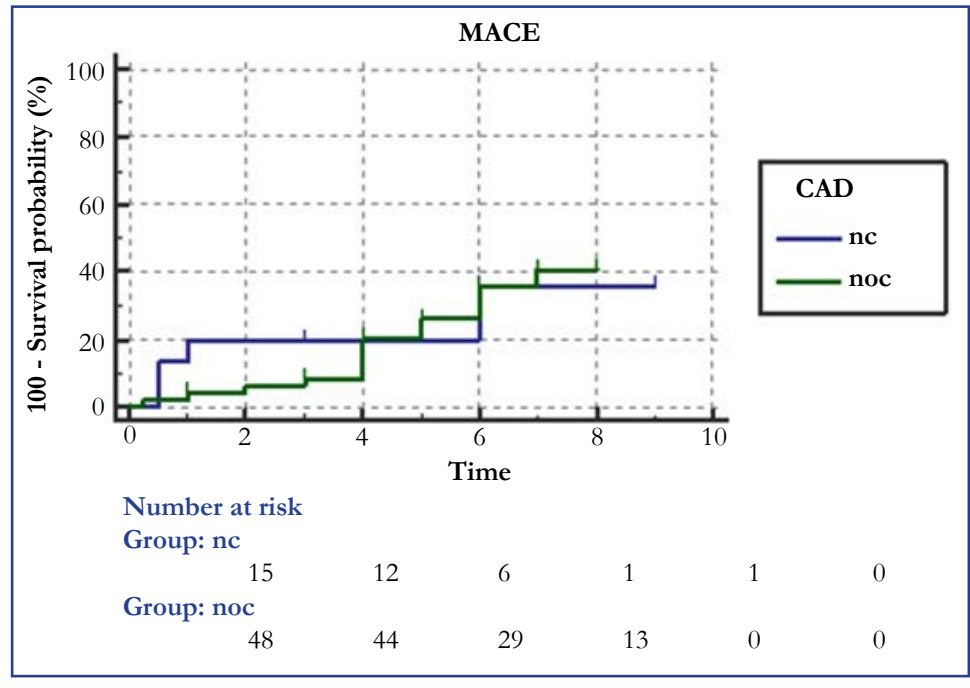

from chest pain, fever or recent $\mathrm{H} / \mathrm{O}$ fever and arrhythmia significantly $(\mathrm{p}<0.05)$ differ in three subgroups. NSTEMI was having the most common category to manifest the symptoms (43.4\% and $60.8 \%$ respectively). Though categorically addressed as non-obstructive atherosclerotic disease, there were some substrate for ACS observed, among which coronary vasospasm being the most common (9\%) followed by myocardial bridge $(8 \%)$. Others were spontaneous dissectionand ectasia (3\% each) and lastly abnormal coronary origin $(2 \%)$ (Figure 1). Another important observation is the prevalence of slow flow which was most common (68\%) in the STEMI subgroup and was statistically significant $\left(x^{2}=6.465, \mathrm{p}\right.$ 0.04) incomparison to NSTEMI/UA. Multiple regression analysis of the variables to predict the major adverse cardiovascular events shows that among above variables only EF has been the significant predictor of MACE (Table 2). The mortality due to all cause death was $13 \%$ among the normal CAG $(<20 \%$ coronary stenosis) group and $10 \%$ in the nonobstructive CAD (20 - 50\% stenosis). Univariate analysis showed that arrhythmia (OR33.8571, CI1.8305-626.2287, p value 0.018) and fever (OR 7.5, CI1.3061- 43.0669, p value- 0.024) were strong and significant predictors of mortality (Table 3 ) and to predict the major adverse events (MACE) Regional wall motion abnormality 
(OR 5.3519, 95\%CI 0.3436-3.5254, p value-0.0382) and the arrhythmia (OR4.5714, 95\%CI 1.45600- 14.3535 , p value 0.0092 ) were the only independent risk factors (Table 4). The Kaplan Meier survival curve analysis shows no significant difference in all cause mortality but significant difference in terms of MACE free survival in these two groups (Figure 2) [The Log-rank test for trend of survival curve difference shows $p$ value 0.04008 and hazard ratio 1.1154 (95\% CI 0.3569 - 3.4856)].

\section{Discussion}

Pasupathy $\mathrm{S}$ et al., in a recent systematic review of the published literature using a $<50 \%$ stenosis threshold for MINOCA(MI with Non-obstructive coronary arteries), has shown the prevalence of this disease to be $6 \%$ [4]. In the large CRUSADE registry, 8.6\% of 38301 patients with NSTEMI did not exhibit obstructive atherosclerosis on angiography [5], a proportion that rose to $10 \%$ in a second report of 55,514 patients [5]. Carmine Pizzi, et al., has demonstrated in the Meta-Analysis of Non-obstructive (NObCAD) Versus Obstructive Coronary Artery Disease (ObCAD) in Acute Coronary Syndrome that as compared with ObCAD subjects, NObCAD patients were significantly younger (6.2 years on average), less likely to be male $(R R=0.77)$, diabetic $(R R=0.57)$, hypertensive $(R R=0.87)$, dyslipidemic $(R R=0.75)[6]$. Though the conventional risk factors like smoking, hypertension, Diabetes Mellitus and dyslipidemia was present in 31\%, 30\%, $15.8 \%$ and $25 \%$ cases in the study population, the feasibility of comparison with the obstructive variety of coronary artery disease is beyond the scope of the study design. However, in our study, there was no statistically significant intra-group difference in normal angiography group vs minor coronary artery disease group in terms of the above risk except hypertension. Gaetano A. Lanza, et al., has described the NSTEACS as not a single disease [7]. It is obvious, however, that this subset is heterogeneous, likely including patients with different mechanisms related to coronary microvascular constriction or spasm. This diagnosis can be suggested by the evidence of slow coronary flow at angiography[ 8]. Importantly, intense coronary microvascular constriction is the likely cause of Takotsubo (apical ballooning) syndrome. Of note, in Planer's study, 16 patients (8.1\%) among those with non-obstructive atherosclerosis were eventually diagnosed with a Takotsubo syndrome, and none had adverse clinical events during follow-up [9]. Leurent et al., [10], who performed CMRI in 107 consecutive MINOCA patients at a mean delay of 6.9 days, reported the following findings with CMRI: myocarditis in $60 \%$, AMI in $16 \%$, Takotsubo cardiomyopathy in $14 \%$, and normal findings in $10 \%$. In the present study, all cause mortality was $13 \%$ in the normal coronary angiography group whereas it was only $10 \%$ in the non-obstructive CAD group (including in-hospital and after discharge both). The presence of arrhythmia and fever played a significant $(p<0.05)$ role i.e. odds ratio 33.85 and 7.5 respectively in predicting the mortality. Gaetano A. Lanza, et al., has shown that 30-day and 1-year all-cause and cardiac mortality rates were similar in patients with and those without obstructive atherosclerosis [8]. At 1 year, all-cause mortality was 3.6\% versus $4.7 \%$, respectively. Of note, patients with non-obstructive atherosclerosis showed a tendency toward a higher rate of noncardiac deaths $(2.1 \%$ versus $1.2 \%$ at 1 year, $P=0.23)$, but a lower rate of recurrent myocardial infarction (1.5\% versus $4.0 \%)$. In our study, the tendency of development of re-infarction and other major vascular event was 10 out of $43(23 \%)$ vs 2 out of $13(15 \%)$ respectively in mild CAD vs normal CAG subgroup. Arrhythmia and decreased LVEF were found to be the two independent predictors for the MACE in the total study population ( $\mathrm{p}$ value $=0.009$ and $<0.0001$ respectively). At 1 year, all-cause mortality was $3.6 \%$ vs $4.7 \%$, respectively, whereas cardiac deaths were $2.4 \%$ vs $2.6 \%$, respectively. Of note, patients with non-obstructive atherosclerosis showed a tendency toward a higher rate of noncardiac deaths $(2.1 \%$ versus $1.2 \%$ at 1 year, $P=0.23)$, but a lower rate of recurrent myocardial infarction (1.5\% versus $4.0 \%)$.

Indeed, a meta-analysis of 18 studies including unselected or stable patients without significant epicardial coronary artery disease reported that coronary events were 6 -fold more frequent in patients with mild $(0-20 \%)$ stenosis and 15 -fold more frequent in patients with moderate stenosis $(20-40 \%)$, when compared with patients with smooth and normal arteriograms. Such a discrepancy, however, may be artificial, because:

(1) Our study was restricted to NObCAD subjects and included a very scarce number of events and was largely underpowered;

(2) heterogeneous conditions were included under the umbrella term of NObACS, which may encompass disparate entities, such as epicardial artery coronary vasospasm, Takotsubo cardiomyopathy, cocaine or other illicit drug abuse, spontaneous coronary dissection, or even acute myocarditis with ACS like presentation.

One limitation of this study is the small sample size. Other limitations include the lack of OCT studies of the coronary arteries considered as the cause of the clinical manifestations and the lack of a control group. Furthermore, we did not perform coronary vasomotor tests. In these patients, the manifestations may have been the result of coronary vasospasms, embolism, or even acute myocarditis.

\section{Key Message}

\section{What is Already Known?}

Non-obstructive coronary artery disease encompasses a heterogeneous group of conditions leading to ACS like features in which no significant obstructive lesions were identified during coronary angiography.

\section{What this Study Adds?}

The results of this study support the concept that non-obstructive CAD is not "insignificant" but rather is associated with a significant and quantifiable risk for cardiovascular morbidity and mortality. This suggests that the traditional dichotomous framework for CAD-useful for characterizing and managing ischemia and cardiac symptoms-should not be applied to ACS and mortality risks inherent in CAD with this entity. Rather, overall CAD extent should be considered a better proxy for both prognosis and management decisions.

\section{Summary and Conclusion}

In conclusion, the absence of atherosclerosis in patients with ACS remains anuncommon but problematic finding in patients undergoing coronary artery angiography. The conventional 
risk factors are not significantly different in subgroup analysis but hypertension. Besides chest pain the other symptoms like dyspnea, arrhythmic symptoms and fever all are most common in NSTEMI.

The all cause mortality does not differ between two groups i.e. normal CAG group and minor CAD group but other fatal cardiovascular events are different between the two. The challenge for researchers and clinicians is now to define the optimal workup for the identification of the specific cause of NSTE-ACS in the individual patient, an essential premise for an appropriate risk stratification and clinical management.

\section{References}

[1]. Benjamin EJ, Blaha MJ, Chiuve SE, Cushman M, Das SR, Deo R, et al. Heart disease and stroke statistics - 2017 update: a report from the American Heart Association. Circulation. 2017 Mar 7;135(10):e146-e603. doi: 10.1161/CIR.0000000000000485. PubMed PMID: 28122885.

[2]. Patel MR, Peterson ED, Dai D, Brennan JM, Redberg RF, Anderson HV, et al. Low diagnostic yield of elective coronary angiography. N Engl J Med. 2010 Mar 11;362(10):886-95. doi: 10.1056/NEJMoa0907272. PubMed PMID: 20220183

[3]. Bugiardini R, Merz CN. Angina with "normal" coronary arteries: a changing philosophy. JAMA. 2005 Jan 26;293(4):477-84. PubMed PMID: 15671433.

[4]. Pasupathy S, Air T, Dreyer RP, Tavella R, Beltrame JF. Systematic review of patients presenting with suspected myocardial infarction and non-obstructive coronary arteries. Circulation. 2015 Mar 10;131(10):861-70. doi:
10.1161/CIRCULATIONAHA.114.011201. PubMed PMID: 25587100.

[5]. Patel MR, Chen AY, Peterson ED, Newby LK, Pollack Jr CV, Brindis RG, et al. Prevalence, predictors, and outcomes of patients with non-ST-segment elevation myocardial infarction and insignificant coronary artery disease: results from the Can Rapid risk stratification of Unstable angina patients Suppress ADverse outcomes with Early implementation of the ACC/AHA Guidelines (CRUSADE) initiative. Am Heart J. 2006 Oct;152(4):641-7. PubMed PMID: 16996828.

[6]. Gehrie ER, Reynolds HR, Chen AY, Neelon BH, Roe MT, Gibler WB, et al. Characterization and outcomes of women and men with non-ST-segment elevation myocardial infarction and nonobstructive coronary artery disease: results from the Can Rapid Risk Stratification of Unstable Angina Patients Suppress Adverse Outcomes with Early Implementation of the ACC/AHA Guidelines (CRUSADE) quality improvement initiative. Am Heart J. 2009 Oct;158(4):688-94. doi: 10.1016/j.ahj.2009.08.004. PubMed PMID: 19781432.

[7]. Pizzi C, Xhyheri B, Costa GM, Faustino M, Flacco ME, Gualano MR, et al. Nonobstructive Versus Obstructive Coronary Artery Disease in Acute Coronary Syndrome: A Meta-Analysis. J Am Heart Assoc. 2016 Dec 16;5(12). PubMed PMID: 27986756.

[8]. Lanza GA, Crea F. Acute Coronary Syndromes Without Obstructive Coronary Atherosclerosis. Circ Cardiovasc Interv. 2014 Jun;7(3):278-81. doi: 10.1161/CIRCINTERVENTIONS.114.001558. PubMed PMID: 24944301.

[9]. Beltrame JF, Limaye SB, Wuttke RD, Horowitz JD. Coronary hemodynamic and metabolic studies of the coronary slow flow phenomenon. Am Heart J. 2003 Jul;146(1):84-90. PubMed PMID: 12851612.

[10]. Leurent G, Langella B, Fougerou C, Lentz PA, Larralde A, Bedossa M, et al. Diagnostic contributions of cardiac magnetic resonance imaging in patients presenting with elevated troponin, acute chest pain syndrome and unobstructed coronary arteries. Arch Cardiovasc Dis. 2011 Mar; 104(3):161-70. doi: 10.1016/j.acvd.2011.01.005. PubMed PMID: 21497305. 